\title{
Inconclusive Findings in Studies of the Link Between Media Coverage of Mass Trauma and Depression in Children
}

\author{
Betty Pfefferbaum ${ }^{1} \cdot$ Phebe Tucker $^{1} \cdot$ Pascal Nitiéma $^{2} \cdot$ Richard L. Van Horn $^{3} \cdot$ Vandana Varma $^{4} \cdot$ Yogesh Varma $^{5}$. \\ Autumn Slaughter ${ }^{6}$. Elana Newman ${ }^{7}$
}

Accepted: 18 January 2022 / Published online: 24 February 2022

(c) The Author(s), under exclusive licence to Springer Science+Business Media, LLC, part of Springer Nature 2022

\begin{abstract}
Purpose of Review This paper reports a review of the empirical research examining the association between mass trauma media contact and depression in children, the factors that may influence the association, and the difficulties encountered in the study of media effects on depression.

Recent Findings All of the included studies assessed general population samples. Pre-COVID-19 research focused primarily on television coverage alone or on multiple media forms including television, while COVID-19 media studies examined various media forms including social media. Most studies used cross-sectional design and non-probability sampling. The review revealed inconclusive findings across studies.

Summary The study of mass trauma media effects on depression in children is complicated by a number of potential confounding factors and by the relatively high prevalence of depression in the general population. Media contact was a relatively minor consideration among other interests in the extant studies which failed to explore numerous issues that warrant attention in future research.
\end{abstract}

Keywords Children and COVID-19 · Children and disasters · Children and terrorism · Depression · Disaster media coverage $\cdot$ Mass trauma media coverage

This article is part of the Topical Collection on Child and Family Disaster Psychiatry

Betty Pfefferbaum

Betty-Pfefferbaum@ouhsc.edu

Phebe Tucker

Phebe-Tucker@ouhsc.edu

Pascal Nitiéma

Pascal.Nitiema-1@ou.edu

Richard L. Van Horn

rvanhorn@ou.edu

Vandana Varma

VVarma@hopechc.org

Yogesh Varma

Yogesh.Varma@intel.com

Autumn Slaughter

aus324@utulsa.edu

Elana Newman

Elana-Newman@utulsa.edu

1 Department of Psychiatry and Behavioral Sciences, College

of Medicine, University of Oklahoma Health Sciences
Center, 920 Stanton L. Young Boulevard, WP 3217, P.O. Box 26901, Oklahoma City, OK 73126-0901, USA

2 Management Information Systems Division, Price College of Business, University of Oklahoma, 307 W. Brooks, Adams Hall 303 Norman, Oklahoma City, OK 73019, USA

3 University of Oklahoma, 920 Stanton L. Young Boulevard, WP 3217, P.O. Box 26901, Oklahoma City, OK 73126-0901, USA

4 Behavioral Health Group, Hope Community Health Center, 7001 Corporate Drive, Suite 120, Houston, TX 77036, USA

5 Intel Corporation, 20475 State Highway TX-249, Houston, TX 77070, USA

6 Department of Psychology, University of Tulsa, 800 South Tucker Drive, Tulsa, OK 74104-3189, USA

7 Dart Center for Journalism and Trauma Research Center, Department of Psychology, 800 South Tucker Drive, Tulsa, OK 74104-3189, USA 


\section{Introduction}

The COVID-19 pandemic is a stark reminder that disasters and other types of mass trauma present frequent and widespread threats to children around the world. Mass trauma events affect children who are directly exposed, those exposed through significant others, and those with no close personal involvement whose only connection is through communication channels and the effects on their community and society. Mass trauma media coverage has the potential to reach large numbers of people including those who are only indirectly or remotely affected as well as those personally involved in an event. A growing literature describes the effects of mass trauma media coverage on children, both those who are directly and indirectly affected by the event. Most of this literature has focused on post-traumatic stress reactions despite the importance of other emotional outcomes such as anxiety and depression which may create an even greater psychological burden [1]. As the second most commonly reported child disaster outcome [2] and a leading and enduring cause of disability worldwide [3], depression is of particular interest.

A recent meta-analysis of studies of mass trauma media contact and depression revealed a small positive association in adult, but not child, samples [4••]. Numerous issues raised in the meta-analysis and other publications warrant greater attention. Specifically, a focused review of the confounds and of methodological issues may clarify aspects of the relationship between mass trauma media contact and depression and help to direct future research on this topic. Further, all of the studies included in the meta-analysis were conducted before the onset of the COVID-19 pandemic, a disaster of unparalleled magnitude resulting in global health, social, economic, and political upheaval. Public health measures implemented during the pandemic resulted in widespread and prolonged closure of schools and home confinement which raised concern about changes in children's lifestyle behaviors (e.g., physical activity and sedentary behavior, diet, sleep) and screen time (i.e., time spent using electronic devices such as television, movies, computers, movies, smartphones, video games).

\section{The Current Review}

The current report describes a review of the empirical literature examining children's depression and mass trauma media contact which was conducted to (1) augment the recent meta-analysis on depression and anxiety [4••] with more recent research including COVID-19 studies and (2) focus specifically on depression and confounding factors. After describing the search process and presenting the results, the discussion focuses on factors that may influence the association between mass trauma media contact and depression, issues related to studying depression as an outcome, and concerns about attributing cause in the association. Issues for future study are identified.

\section{Methods}

An initial literature search to identify empirical research meeting inclusion criteria was conducted in March 2020 using ERIC, MEDLINE, PsycINFO, PTSDpubs, and Social Work Abstracts databases. Search terms were selected to locate references on the association between depression and/or anxiety outcomes and consumption of media coverage (broadcast, internet, magazine, newspaper, print, radio, social media, television, TV, web) of mass trauma (bombing, disaster, earthquake, fire, flood, hurricane, mass shooting, political conflict, September 11, terrorism, terrorist incident, tornado, tsunami, war). The search was restricted to publications in peer-reviewed journals and to English language sources, with no restrictions on participant age nor publication date. To identify publications since the initial search, another search was conducted in March 2021 using the same databases with additional search terms to capture literature on epidemics, pandemics, and COVID-19 as well as more recent publications on other mass trauma events. Studies were included in this review if: (1) children were assessed, (2) media coverage related to mass trauma was assessed, (3) contact with media coverage was systematically measured (e.g., dose of contact such as number of hours spent watching disaster television coverage, content of disaster coverage consumed), (4) depression outcomes were systematically assessed, and (5) the association between media contact and depression was estimated with a statistical method. Together the searches, and articles known to the authors, yielded a total of 12 articles on media contact and children's depression outcomes in the context of mass trauma (see Fig. 1).

\section{Descriptive Findings in the Literature}

Empirical studies on children's depression reactions to disaster media coverage are described below (see Table 1 for a summary of the 12 studies $[5,6,7,8 \bullet \bullet, 9,10,11 \bullet, 12,13$, $14 \bullet \bullet, 15 \bullet \bullet, 16]$ included in this review).

\section{Events, Samples, and Participant Exposure}

The studies in this review of media contact and depression examined children in the context of terrorism, especially

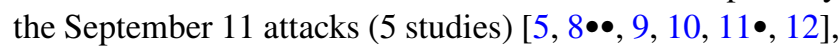
along with a study in Israel [6] and of the global COVID-19 
Fig. 1 Flowchart of the literature search

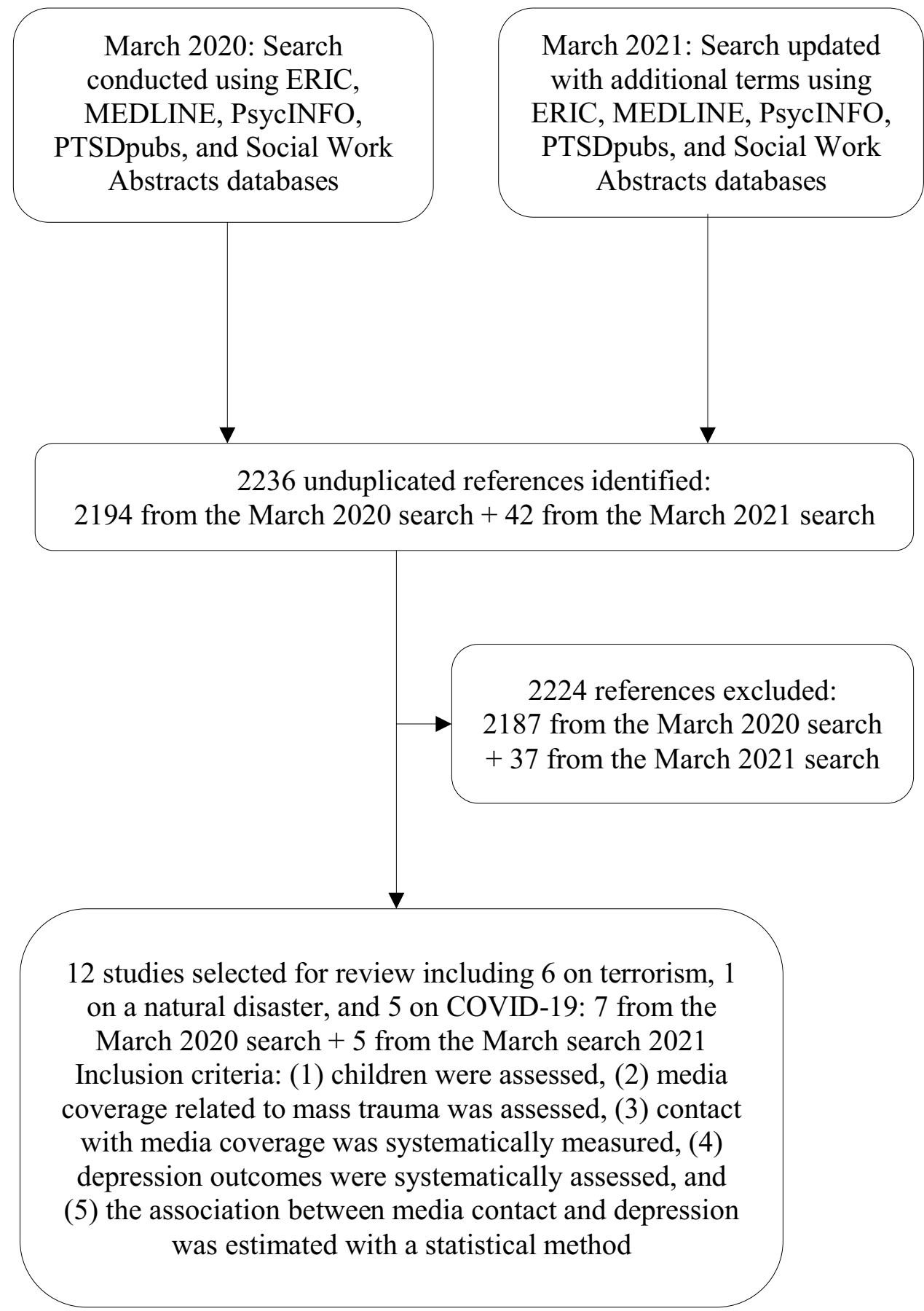

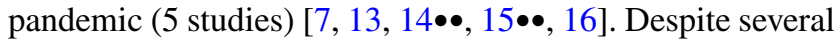
major natural disasters (e.g., 2004 Indian Ocean earthquake and tsunami, the 2011 Great East Japan earthquake and tsunami) that attracted worldwide attention through the media, only one study was conducted in the context of a natural disaster - the 2008 Sichuan earthquake in China [11•] (see Table 1). All of these events had wide-reaching communal effects on general populations as well as on those who were directly exposed and personally involved. None of the 12 studies in this review analyzed media contact in relationship to participants' event exposure.

All of the pre-COVID-19 studies in the current review assessed samples derived from general populations in schools, the disaster community, and distant communities rather than samples chosen for specific exposures (e.g., directly-exposed children); some of these samples included directly exposed children and/or children exposed through the experiences of family members or others as well as those 


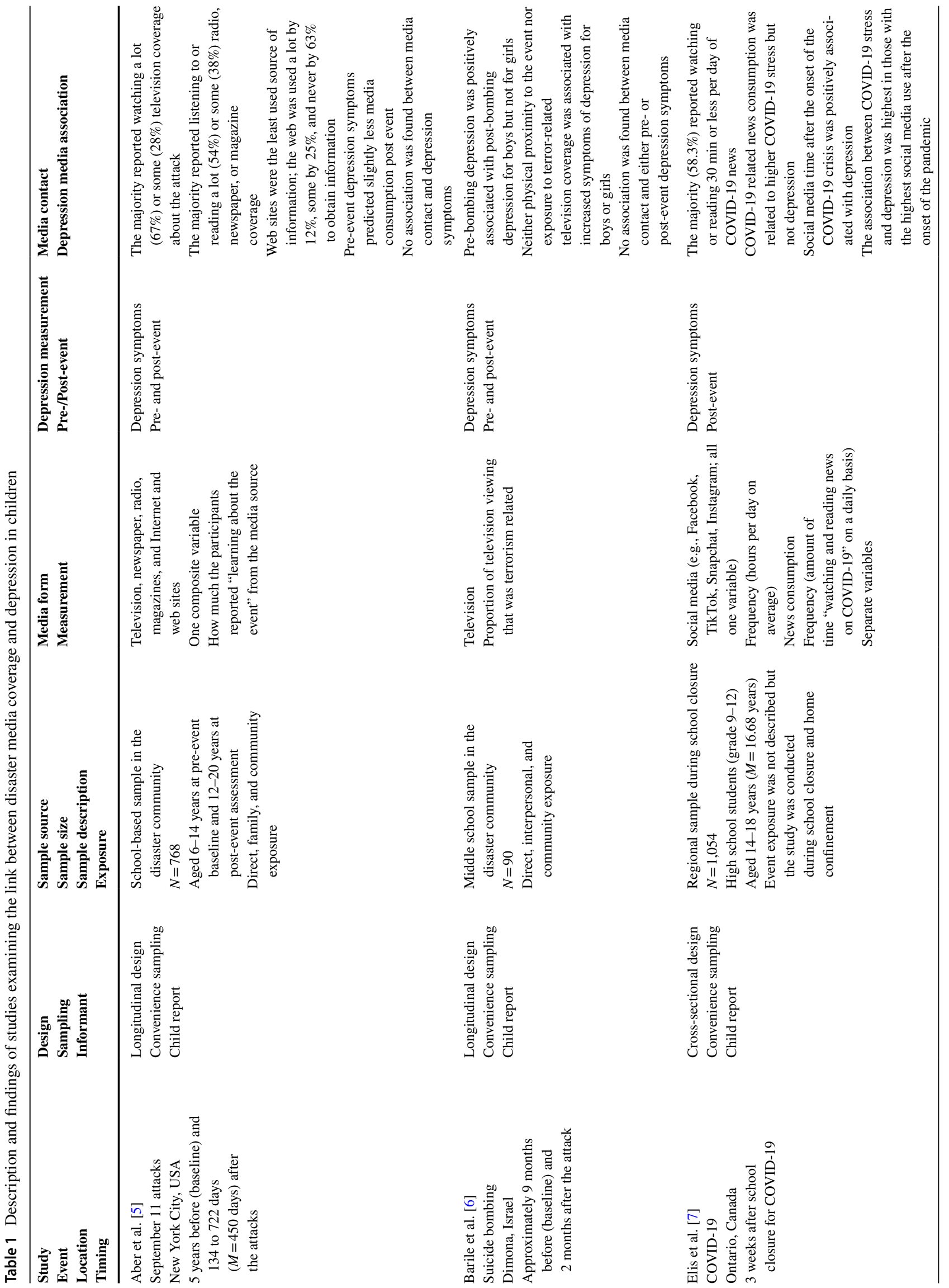




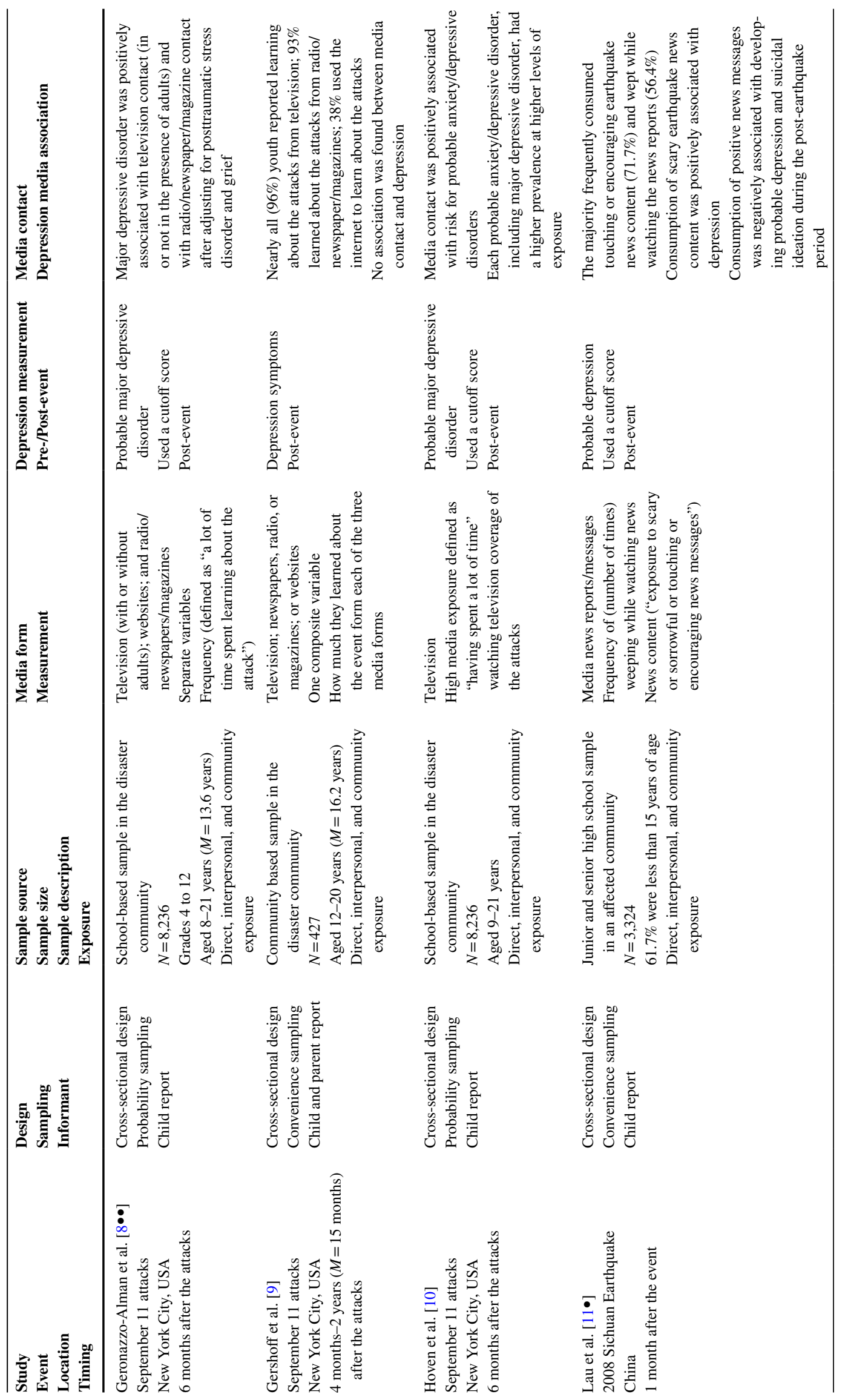




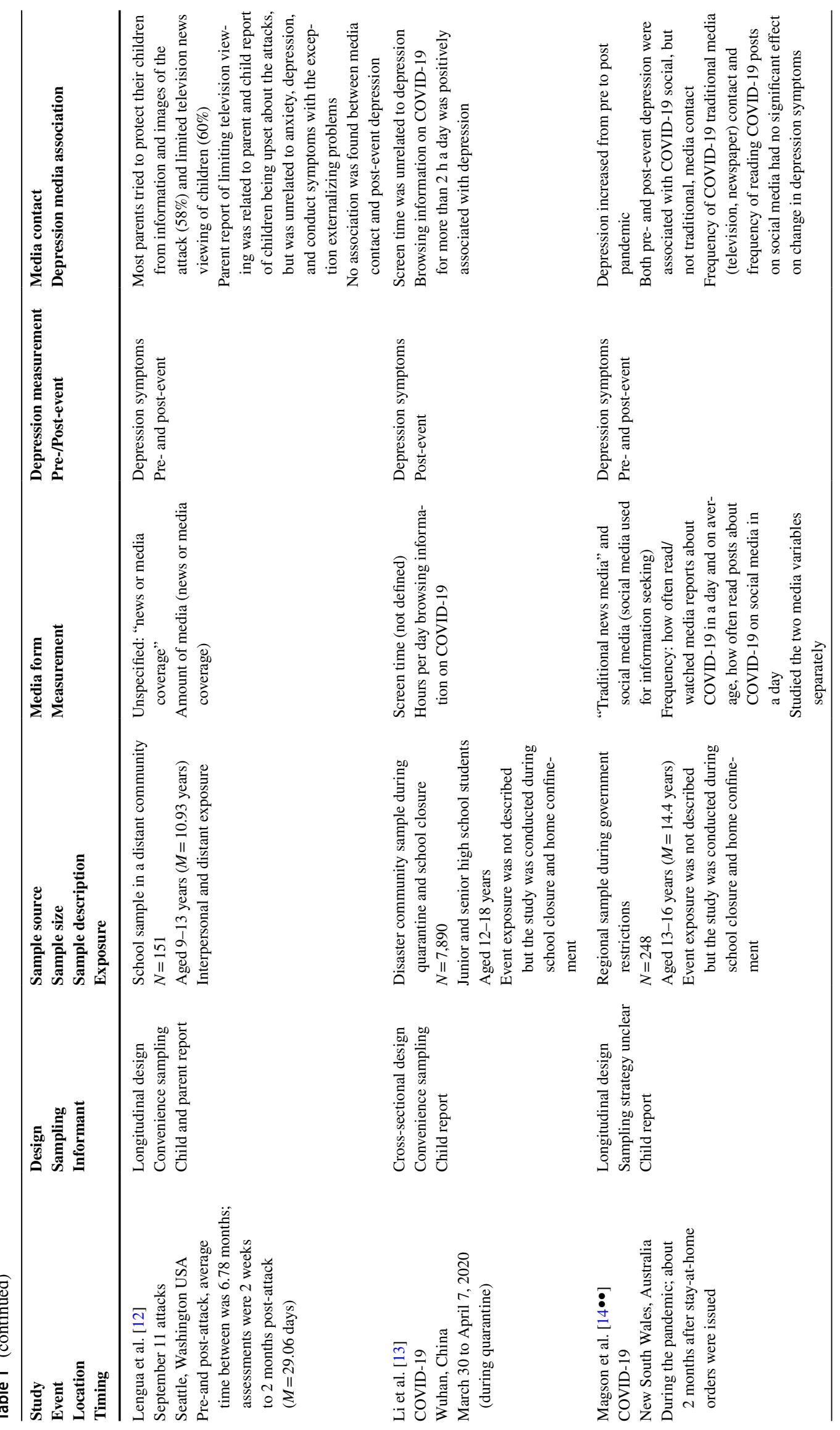




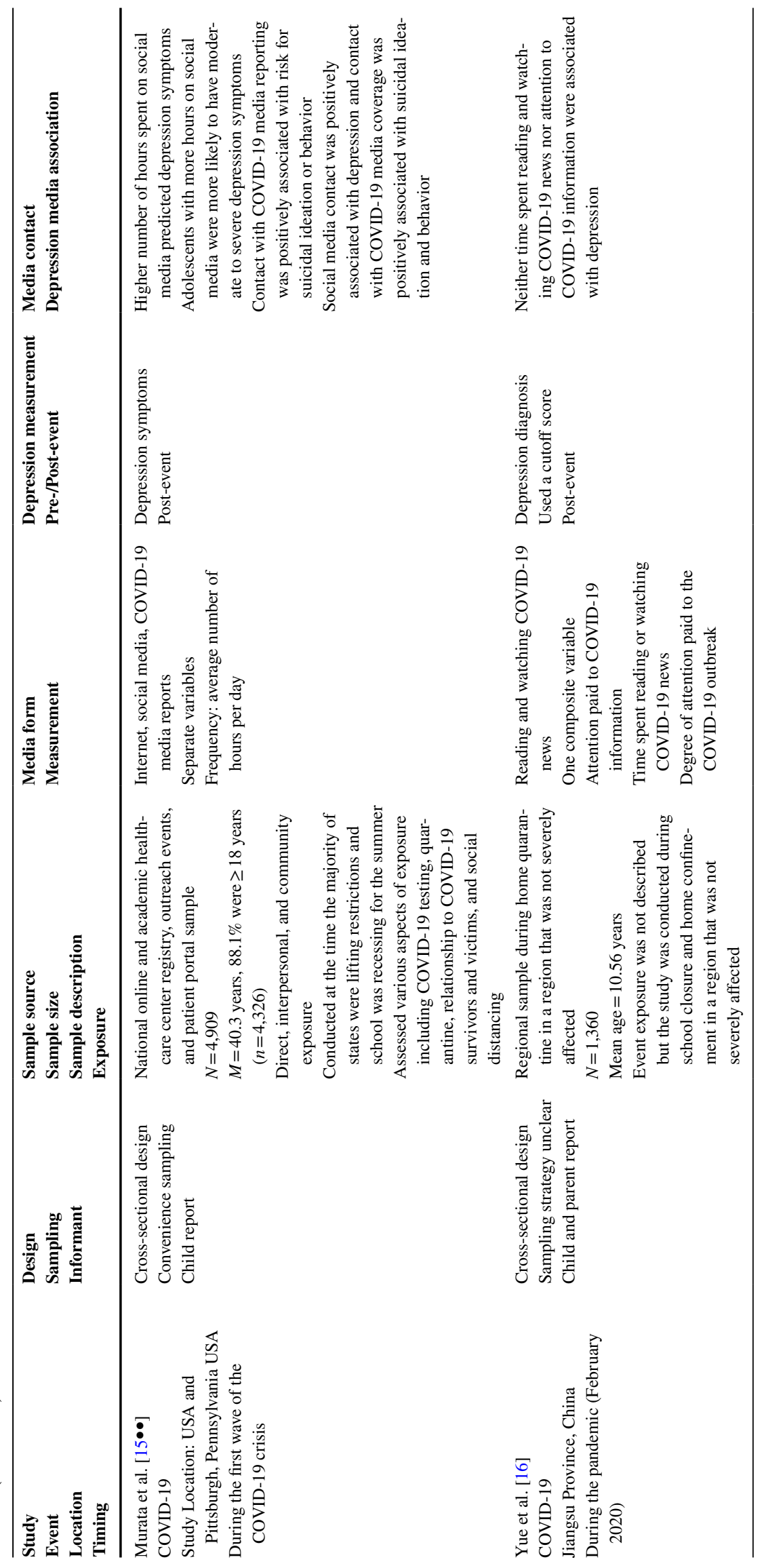


whose only contact was through community and media channels $[5,6,8 \bullet \bullet, 9,10,11 \bullet, 12]$. Most of these studies were conducted in the community where the event occurred $[5,6,8 \bullet \bullet, 9,10]$. One September 11 study was conducted in Seattle, WA, USA [12], far from the communities directly attacked. The study of the Sichuan earthquake was conducted in Chengdu, China, approximately $90 \mathrm{~km}$ from the epicenter of the earthquake [11•] (see Table 1).

Four of the five COVID-19 studies assessed participants in regions where public health restrictions were in place; none of these four studies described explicit measures of

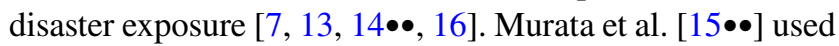
a mixed recruitment strategy including a national online approach across the USA and advertisement through an academic healthcare center registry, outreach events, and patient portal. Their study, conducted at a time when some states were lifting public health restrictions, assessed various aspects of exposure including COVID-19 testing, quarantine, relationship with COVID-19 survivors and victims, and social distancing [15••] (see Table 1).

\section{Media Forms and Extent of Contact}

The pre-COVID-19 research assessed children's reactions to either television coverage alone $[6,10]$ or multiple media forms including television $[5,8 \bullet \bullet, 9]$ or did not specify the media form $[11 \bullet, 12]$. While several studies assessed inter-

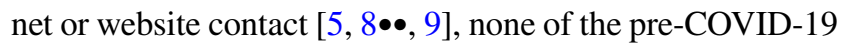
studies specifically queried social media contact. In contrast, COVID-19 research examined various media forms including social as well as traditional media (see Table 1). The purpose of social media use (e.g., information gathering, socialization, entertainment) varied across studies. While most of the 12 studies measured the amount of media coverage consumed (e.g., amount of time, frequency of contact, proportion of all media contact), some studies assessed how much or how frequently participants reported learning about

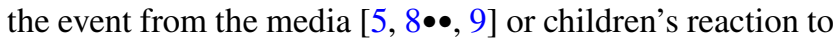
coverage [11•] instead. One COVID-19 study queried how much time participants spent browsing for information about coronavirus [13], and one asked about participants' attention to information about COVID-19 [16], but neither specified that these questions referred exclusively to media coverage (see Table 1).

\section{Depression Outcomes}

Of the 12 studies included in this review, four used a cutoff score to analyze probable post-event major depressive disorder in association with media contact $[8 \bullet \bullet, 10,11 \bullet, 16]$. Others used depression symptoms in their media analyses $[5,6,7,9,12,13,14 \bullet \bullet, 15 \bullet \bullet$. Four studies reported data on pre-event depression symptoms $[5,6,12,14 \bullet \bullet$ (see Table 1).

\section{Pre-Event Depression}

Aber et al. [5] found that pre-event baseline depression symptoms predicted slightly less media consumption after the September 11 attacks in their adolescent sample. In their study of Israeli children after a suicide bombing, Barile et al. [6] found relatively low levels of depression both pre- and post-event, with lower post-event than pre-event depression scores and no association between pre-event depression and media contact. In their distant sample of children assessed before and after the September 11 attacks, Lengua et al. [12] found that both child- and parent-reported depression decreased significantly at their first post-event assessment 2 weeks to 2 months after the September 11 attacks relative to the pre-event assessment and increased again at followup 6 months after September 11. They did not examine the association between media contact and pre-event depression and found no association between media contact and post-event depression [12]. Magson et al. [14••] found that depression increased during the COVID-19 pandemic relative to an earlier assessment; that both pre- and post-event depression were associated with COVID-19 social, but not traditional, media contact; and that change in depression was not associated with consumption of either COVID-19 traditional or social media coverage (see Table 1).

\section{Associations Between Media Contact and Depression}

The results of the pre-COVID-19 studies included in the current review were inconsistent with respect to the association between media contact and depression. Two terrorism studies found an association between probable major depressive disorder and media contact $[8 \bullet \bullet, 10]$ while four studies found no association between depression symptoms and media contact $[5,6,9,12]$. In their study of grief, depression, and posttraumatic stress in New York City children after the September 11 attacks, Geronazzo-Alman et al. [8・日 found that major depressive disorder was associated with media variables in a model adjusted for grief and posttraumatic stress disorder (PTSD). The study of the Sichuan earthquake found that contact with frightening news content was associated with probable depression, while positive news messages protected against probable depression and suicidal ideation [11•] (see Table 1).

The COVID-19 studies also were inconsistent with respect to the association between media contact and depression. $\mathrm{Li}$ et al. [13] found that screen time, which was not defined, was not associated with depression symptoms, while browsing COVID-19 information for more than $2 \mathrm{~h}$ a day was 
associated with depression symptoms. Among other studies that examined COVID-19 media consumption, one found that greater contact with COVID-19 media reporting was associ-

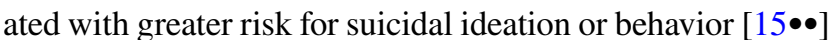
and two found no association between COVID-19 news consumption and depression [7, 16]. Magson et al. [14••] found an association between depression symptoms and consumption of COVID-19 social media, but not COVID-19 traditional media, with no association between change in depression symptoms and contact with either media form. Of the other COVID-19 studies that examined social media contact $[7,15 \bullet \bullet]$, one found that both social media time and virtual time with friends were positively associated with depression symptoms [7] and one, which did not indicate the content of, or purpose for using, social media, found an association between social media and depression symptoms [15••] (see Table 1).

\section{Methodological Review}

The methodological rigor of the studies varied greatly. Most studies used cross-sectional design $[7,8 \bullet \bullet, 9,10,11 \bullet, 13$, $15 \bullet \bullet, 16]$, with only four longitudinal studies that measured depression symptoms before and after the event $[5,6,12$, $14 \bullet \bullet]$. Only two papers used probability sampling $[8 \bullet \bullet, 10]$. Children $[5,6,7,8 \bullet \bullet, 10,11 \bullet, 13,14 \bullet \bullet, 15 \bullet \bullet]$ or children and their parents $[9,12,16]$ were the informants in all 12 studies. In the longitudinal September 11 study by Aber et al. [5], children and teachers were informants at the baseline assessment prior to the event, but only students reported on pre-event depression. As noted, eight studies used depression symptoms in the analysis of media contact $[5,6,7,9$, $12,13,14 \bullet \bullet, 15 \bullet \bullet$, while four used a clinical cutoff for depression $[8 \bullet \bullet, 10,11 \bullet, 16]$. With respect to contact with various media forms, some studies examined only television coverage $[6,10]$, some used separate variables for each media form examined $[7,8 \bullet \bullet, 14 \bullet \bullet, 15 \bullet \bullet]$, others used a composite variable to examine more than one media form [5, $9,16]$, and others did not specify the media forms examined $[11 \bullet, 12,13]$ (see Table 1). Further, many of the studies used only one or two media variables which varied considerably across the research, making it difficult to consolidate the results. None of the studies used depression measures that queried whether symptoms were related to the event or to coverage of the event.

\section{Discussion}

The inconclusive findings related to the association between media contact and depression across both pre-COVID-19 and COVID-19 studies align with the results of a recent pre-pandemic meta-analysis that found no association between media contact and depression in children in the

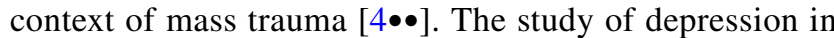
the context of mass trauma is complicated by a number of confounding factors that may influence the associationincluding those related to the mass trauma event and context, to aspects of the media coverage and contact, and to the children and their exposure-and by the relatively high prevalence of depression symptoms and major depressive disorder in the general population $[17,18]$. This discussion considers these confounding factors, issues related to the measurement of depression outcomes, and causality.

\section{Potential Confounding Factors in the Association Between Media Contact and Depression}

Among potential confounding factors that may influence children's depression reactions to media coverage are characteristics of the event and context; aspects of media coverage and consumption; and the child's prior and/or subsequent experiences, psychiatric history, and event exposure. While not meant to constitute an exhaustive review, some of these factors are considered below along with methodological limitations in the extant research.

\section{Characteristics of the Event and Context}

A host of event characteristics (e.g., magnitude, probability) are likely to influence children's reactions to media coverage. In their meta-analysis of adult and child studies of depression outcomes associated with mass trauma media contact, Pfefferbaum et al. [4••] found a significant positive association between depression and coverage of specific incidents but not for chronic stressors (e.g., ongoing political conflict), perhaps because of the small number of studies of chronic situations. Of note, the Israeli study by Barile et al. [6] found no association between depression and media coverage of a suicide bombing in children, but the event occurred in the context of ongoing political violence and thus shared features of chronic stressors. It may be that specific aspects of enduring stressors or of media coverage and contact in these environments are responsible for these outcomes. For example, a recent review of Israeli child terrorism media studies found inconclusive results related to the association between media contact and a variety of psychological outcomes perhaps, in part at least, due to the nature of coverage in a region with a long history of political conflict [19]. Evidence suggests that media coverage in Israel typically delivers event-related information, political commentary, content on expected psychological reactions, and advice and support for mental health recovery [20]. The COVID-19 pandemic shares some characteristics with chronic political violence 
including, among other things, the ongoing threat with no foreseeable end, intense media coverage, and societal effects that alter numerous aspects of daily life. COVID-19 coverage has been characterized by incomplete information, inconsistent messages, and copious misinformation often reflecting highly politicized agendas delivered by unqualified sources [21]. This coverage has generated confusion and sowed mistrust in government and public health authorities, likely influencing the reactions of many consumers. These considerations demonstrate the difficulties in identifying and examining contextual factors that influence the association between media contact and emotional outcomes.

\section{Aspects of Media Coverage and Contact}

A number of aspects of media coverage (e.g., content, images) and contact (e.g., media form, motivation for consumption, context) have the potential to influence the association between children's consumption and depression outcomes. Prior to the COVID-19 pandemic, television coverage was the predominant media form examined in the child disaster media literature. Children's use of social media has gained popularity over the years, but social media contact, as currently conceptualized, was seldom covered in pre-COVID-19 research with only three September 11 studies assessing depression in association with consumption of multiple media forms including internet and/or website

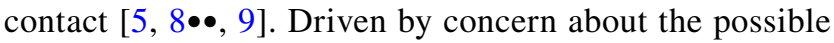
deleterious effects of increased screen time during extended home confinement in addition to concern about media content, COVID-19 studies examined numerous media forms including various social media platforms.

The pre-COVID-19 child mass trauma research generally used media contact variables linked to the event by querying event-related media consumption. As noted above, this was not universally true of the COVID-19 research assessing screen time [13] or social media [7, 15••] or internet [15••] use which did not query children's motivation for media consumption $[7,13,15 \bullet \bullet]$. The failure to distinguish media contact related to COVID-19 from contact for social, entertainment, or other purposes clouded the findings and seriously limited the interpretation of the results related to the association between media contact and depression. The inconclusive results regarding the association of media contact with depression are not surprising given the great diversity in questions used to assess media coverage and media consumption and the fact that some COVID-19 studies did not assess children's contact with consumption of media coverage focused on the pandemic. A discussion of the association between social media contact and depression in children in the context of mass trauma is beyond the scope of this review. Such a review should consider, among other things, the multiple forms of social media and the diverse purposes of children's social media use, the various potential determinants of children's social media behavior, and both positive and negative outcomes associated with social media contact.

\section{Participant Characteristics: Personal Vulnerabilities and Event Exposure}

A host of individual factors, including demographic and vulnerability factors (e.g., prior psychiatric disorders, previous trauma exposure), influence children's disaster reactions and may also influence their reactions to media coverage. Vulnerability factors have not been well examined in the child disaster media literature. Of the four studies that assessed pre-event depression [5, 6, 12, $14 \bullet \bullet$, only two examined its association with post-event media contact $[5,6]$. One found that pre-event depression symptom severity predicted slightly less post-event media consumption [5] and one found no association between pre-event depression symptoms and post-event media contact [6]. The results of the COVID-19 study by Magson et al. [14••] suggested that the patterns of the associations between media contact and depression symptoms before and during the pandemic were similar (i.e., at both time periods, there was no association with traditional media contact but a positive association with social media contact) and that the pandemic did not influence the pattern of association between depression and media consumption. Future research should further examine pre-event depression and other child vulnerabilities that may influence the association between media contact and depression.

The mechanisms by which media contact affects children likely depend, in part at least, on their specific trauma experiences. A meta-analysis of posttraumatic stress reactions in adult and child terrorism media studies, which found a greater effect for media contact in studies of distant samples [22], suggested that media consumption may constitute a primary source of fear and other reactions in those who are not directly exposed to an event. In directly exposed children, media coverage may rekindle trauma memories and generate arousal, but personally experienced event trauma is likely to dwarf the influence of media coverage in determining the nature and degree of psychological outcomes. Evidence suggests that while directly exposed individuals are likely to develop posttraumatic stress, those with indirect exposure are more likely to experience depression [23•]. All of the studies included in the current review examined general population samples rather than samples of children selected because of their 
particular personal involvement (e.g., direct exposure, interpersonal exposure). Unfortunately, none of the studies examined the influence of the child's event exposure on the association between media consumption and depression in the participating children. This issue should be addressed in future research.

\section{The Depression Outcome}

Studying depression in the context of mass trauma is complicated by a number of issues. First is the high number of cases of depression outside the context of mass trauma in general. Second are measurement issues involving the assessment of the prevalence and incidence of depression and the choice of diagnosis or symptoms as an outcome measure.

\section{Depression Rates and the Assessment of Prevalence and Incidence}

Most of the mass trauma media research fails to consider the fact that depression observed during or after a mass trauma may not be caused by the event and/or may even pre-date the event. Only four studies in this review reported pre-event depression measures $[5,6,12,14 \bullet \bullet]$. Studies that examined pre-event depression symptoms suggest that it may have an effect on media behavior [5, 14••] though the findings regarding the association between pre-event depression and media contact were inconsistent $[5,6,14 \bullet \bullet]$. Magson et al. [14••] found that the change in depression scores from prepandemic to during the pandemic was not associated with media contact. Like most mass trauma media studies that have assessed post-event depression outcomes, none of the analyses distinguished between prevalence (i.e., the proportion of children with depression symptoms or depressive disorder during the post-event period) and incidence (i.e., the proportion of children who developed new depression symptoms or depressive disorder during the post-event period), making it impossible to determine if the depression outcomes were new since the event, and possibly caused by it, or if they were present before the event and thus represented relapses or ongoing symptoms or disorders rather than new symptoms or disorders.

\section{Symptoms and Diagnosis}

Like child disaster research on depression in general, most of the studies included in this review measured symptoms rather than diagnoses. Distress is ubiquitous in post-event settings, but, for most, it does not endure or develop into psychiatric disorders. Thus, finding an association between media contact and depression symptoms, rather than a diagnostic outcome, may not be clinically meaningful.

\section{Cause and Effect}

Mass trauma events like those examined in the studies included in this review-the September 11 attacks, a suicide bombing in the context of ongoing political violence, a massive earthquake, and the global COVID-19 pandemicaffect large numbers of people with sometimes extensive and enduring community and societal effects. Depression symptoms are not unlikely following mass trauma given the many burdens and obstacles created by these events including personal and communal loss and trauma, property damage, disrupted services and support networks, and widespread distress. Children may turn to media coverage and/or social media to obtain information or to cope. Thus, studies that found an association between media contact and depression raise the issue of causation. It is possible that media consumption leads to depression or that depressed children seek media coverage or turn to social media. Concerns about the use of social media-particularly social media used for social connections - and the cause and effect issue were prominent during the extended period of home confinement in the COVID-19 pandemic. For example, depressed children may have used social media connections with friends to alleviate depression, thereby generating a positive association, or social media contact with friends may have lessened depression perhaps resulting in a negative association. Indeed, most of the extant research on the association between social media use and depression symptoms in youth has assessed correlations rather than establishing a causal relationship and has revealed inconsistent small associations that are not likely of clinical or practical significance [24].

Unlike PTSD, depression is not conditioned on exposure to trauma. Thus, symptoms need not be anchored in the event as reflected in the studies in this review, none of which asked participants if their depression symptoms were either related to or new since the event or to media coverage of the event. A link to trauma is not a required element in the assessment of depression, a subjectively reported link would not be conclusive in distinguishing prevalence and incidence, and an association between media contact and depression would not establish a causal relationship. Nonetheless, asking participants if they perceived their depression symptoms to be associated with the trauma or media coverage would provide useful information when assessing reactions to disaster media coverage. Thus, future research anchoring depression in the event and/or in media coverage of the event is warranted. 


\section{Conclusions}

Relatively little empirical research has examined the association between media contact and depression in children in the context of mass trauma, with most studies examining terrorist events and the COVID-19 pandemic and only one natural disaster study. The review of this research, which yielded inconclusive results, highlighted the difficulties encountered in the study of media effects on depression. Taken as a whole, the extant literature, both pre-pandemic and COVID-19 publications, reported findings from research that was not focused primarily on media effects but examined media contact as a relatively minor consideration among other interests. Thus, the exploration of the topic was relatively superficial and left numerous issues unaddressed. Concern about children's increased screen time during COVID-19 pandemic restrictions generated studies to augment the relatively sparse disaster media effects literature on depression in children, but the new pandemic research has introduced additional theoretical and methodological complexities in the study of this topic. For example, while COVID-19 studies added a focus on social media contact, it raised questions about the purpose of social media use (e.g., to obtain and exchange information on COVID-19, to engage in social interaction, to be entertained) and about potential differences among various social media platforms as well as other factors related to individual children and the context of their media behavior. Future research specifically examining social media contact and depression in children in the disaster context and future reviews consolidating the results of this research are warranted.

Key among the complexities in the study of the association between media contact and depression is the relatively high prevalence of depression in the general population. Major mass trauma events and their secondary effects (e.g., disrupted services) tend to permeate the post-event environment, making it difficult to distinguish media contact and other channels of information and affect. The extant research has not established a causal relationship between depression and media consumption, raising the possibility that a number of factors may influence, or be the primary determinant of, the association. Furthermore, none of the studies included in this review was focused primarily on media effects, and all used a limited number of variables to examine aspects of media coverage and media consumption. These considerations serve as a reminder that multiple determinants interact to influence children's outcomes. Unfortunately, while some of these determinants have been identified, none has been adequately examined. The field would be well served by methodologically rigorous studies that focus specifically on the numerous intricate and dynamic media issues and on the many individual and contextual factors that influence the association between media consumption and depression.
Acknowledgements The editors would like to thank Dr. Namik Kirlic for taking the time to review this manuscript.

\section{Compliance with Ethical Standards}

Conflict of Interest Betty Pfefferbaum, Phebe Tucker, Pascal Nitiéma, Richard L. Van Horn, Vandana Varma, Yogesh Varma, Autumn Slaughter, and Elana Newman each declare no potential conflicts of interest.

Human and Animal Rights and Informed Consent This article does not contain any studies with human or animal subjects performed by any of the authors.

\section{References}

Papers of particular interest, published recently, have been highlighted as:

- Of importance

$\bullet$ Of major importance

1. Moss WJ, Ramakrishnan M, Storms D, Siegel AH, Weiss WM, Lejnev I, Muhe L. Child health in complex emergencies. Bull World Health Org. 2006;84(1):58-64. https://doi.org/10.2471/blt. 04.019570 .

2. Wang CW, Chan CLW, Ho RTH. Prevalence and trajectory of psychopathology among child and adolescent survivors of disasters: a systematic review of epidemiological studies across 1987-2011. Soc Psychiatry Psychiatr Epidemiol. 2013;48:1697720. https://doi.org/10.1007/s00127-013-0731-x.

3. Gore FM, Bloem PJN, Patton GC, Ferguson J, Joseph V, Coffey $\mathrm{C}$, et al. Global burden of disease in young people aged 10-24 years: a systematic analysis. Lancet. 2011;377:2093-102. https:// doi.org/10.1016/s0140-6736(11)60512-6.

4.•• Pfefferbaum B, Nitiéma P, Newman E. The association of mass trauma media contact with depression and anxiety: a meta-analytic review. J Affect Dis Rep. 2021;3:100063. https://doi.org/10.1016/j. jadr.2020.100063. This meta-analysis of studies of mass trauma media contact and depression revealed a small positive association in adult, but not child, samples and a significant positive association between depression and coverage of specific incidents but not for chronic stressors (e.g., ongoing political conflict), perhaps because of the small number of studies of chronic situations.

5. Aber JL, Gershoff ET, Ware A, Kotler JA. Estimating the effects of September 11 and other forms of violence on the mental health and social development of New York City's youth: a matter of context. Appl Dev Sci. 2004;8(3):111-29. https://doi.org/ 10.1207/s1532480xads0803 2.

6. Barile JP, Grogan KE, Henrich CC, Brookmeyer KA, Shahar G. Symptoms of depression in Israeli adolescents following a suicide bombing: the role of gender. J Early Adolesc. 2012;32(4):502-15. https://doi.org/10.1177/0272431611400317.

7. Ellis WE, Dumas TM, Forbes LM. Physically isolated but socially connected: psychological adjustment and stress among adolescents during the initial COVID-19 crisis. Can J Behav Sci. 2020;52(3):177-87. https://doi.org/10.1037/cbs0000215.

8.•• Geronazzo-Alman L, Fan B, Duarte CS, Layne CM, Wicks J, Guffanti G, et al. The distinctiveness of grief, depression, and posttraumatic stress: lessons from children after 9/11. J Am Acad Child Adolesc Psychiatry. 2019;58(10):971-82. https://doi.org/ 10.1016/j.jaac.2018.12.012. This study of grief, depression, 
and posttraumatic stress in a representative sample of New York City school children, assessed six months after the September 11, 2001, attacks, found that major depressive disorder was associated with media contact variables in a model adjusted for grief and posttraumatic stress disorder.

9. Gershoff ET, Aber JL, Ware A, Kotler JA. Exposure to 9/11 among youth and their mothers in New York City: enduring associations with mental health and sociopolitical attitudes. Child Dev. 2010;81(4):1142-60. https://doi.org/10.1111/j.14678624.2010.01459.x.

10. Hoven CW, Duarte CS, Lucas CP, Wu P, Mandell DJ, Goodwin RD, et al. Psychopathology among New York City public school children 6 months after September 11. Arch Gen Psychiatry. 2005;62:545-52. https://doi.org/10.1001/archpsyc.62.5.545.

11.• Lau JTF, Yu X, Zhang J, Mak WWS, Choi KC, Liu WWS, et al. Psychological distress among adolescents in Chengdu, Sichuan at 1 month after the 2008 Sichuan earthquake. J Urban Health. 2010;87(3):504-23. https://doi.org/10.1007/s11524-010-94473 . This study assessing secondary school students living in a community affected by the 2008 Sichuan earthquake found that contact with frightening news media content was associated with probable depression and that positive news messages protected against probable depression and suicidal ideation.

12. Lengua LJ, Long AC, Smith KI, Meltzoff AN. Pre-attack symptomatology and temperament as predictors of children's responses to the September 11 terrorist attacks. J Child Psychol Psychiatry. 2005;46(6):631-45. https://doi.org/10.1111/j.1469610.2004.00378.x.

13. Li W, Zhang Y, Wang J, Ozaki A, Wang Q, Chen Y, Jiang Q. Association of home quarantine and mental health among teenagers in Wuhan, China, during the COVID-19 pandemic. JAMA Pediatr. 2021;175(3):313-6. https://doi.org/10.1001/jamapediatrics.2020. 5499.

14.• Magson NR, Freeman JYA, Rapee RM, Richardson CE, Oar EL, Fardouly J. Risk and protective factors for prospective changes in adolescent mental health during the COVID-19 pandemic. J Youth Adolesc. 2021;50;44-57. https://doi.org/10.1007/s10964020-01332-9. This longitudinal study of adolescents found that depression increased after implementation of COVID-19 government restrictions relative to a pre-pandemic assessment; that both pre- and post-event depression were associated with COVID-19 social, but not traditional, media contact; and that change in depression was not associated with consumption of either COVID-19 traditional or social media coverage.

15.•• Murata S, Rezeppa T, Thoma B, Marengo L, Krancevich $\mathrm{K}$, Chiyka E, et al. The psychiatric sequelae of the COVID19 pandemic in adolescents, adults, and health care workers. Depress Anxiety. 2021;38:233-46. https://doi.org/10.1002/da. 23120. This study assessing adolescents in the United States at a time when some states were lifting COVID-19 public health restrictions found that social media contact was associated with depression symptoms and that contact with COVID-19 media reporting was positively associated with risk for suicidal ideation or behavior.

16. Yue J, Zang X, Le Y, An Y. Anxiety, depression and PTSD among children and their parent during 2019 novel coronavirus disease (COVID-19) outbreak in China. Curr Psychol. 2020;14:1-8. https://doi.org/10.1007/s12144-020-01191-4.

17. Kessler RC, Berglund P, Demler O, Jin R, Koretz D, Merikangas $\mathrm{KR}$, et al. The epidemiology of major depressive disorder. Results from the National Comorbidity Survey Replication (NCS-R). JAMA. 2003;289(23):3095-3105. https://doi.org/10.1001/jama. 289.23.3095

18. World Health Organization. Depression and other common mental disorders. Global health estimates. Geneva, Switzerland. 2017. Available from https://apps.who.int/iris/bitstream/handle/ 10665/254610/WHO-MSD-MER-2017.2-eng.pdf?sequence $=1$ \&isAllowed $=\mathrm{y}$.

19. Pfefferbaum B, Tucker P, Newman E, Nelson SD, Nitiéma P, Pfefferbaum RL. Terrorism media effects in youth exposed to chronic threat and conflict in Israel. Curr Psychiatry Rep. 2019;21:28. https://doi.org/10.1007/s11920-019-1005-4.

20. Tatar M, Amram S. Israeli adolescents' coping strategies in relation to terrorist attacks. Br J Guid Counsel. 2007;35(2):163-73. https://doi.org/10.1080/03069880701256569.

21. Editorial. The COVID-19 infodemic. Lancet Infect Dis. 2020;20(8):875. https://doi.org/10.1016/S1473-3099(20)30565-X.

22. Houston JB. Media coverage of terrorism: a meta-analytic assessment of media use and posttraumatic stress. J Mass Commun Q. 2009;86(4):844-61. https://doi.org/10.1177/107769900908600408.

23. North CS, Baron D, Chen AF. Prevalence and predictors of postdisaster major depression: convergence of evidence from 11 disaster studies using consistent methods. J Psychiatr Res. 2018;102:96-101. https://doi.org/10.1016/j.jpsychires.2017.12. 013 . This study of predictors of post-disaster major depression using composite databases from multiple disasters found that post-disaster major depression was independently associated with pre-existing major depression, indirect exposure to disaster trauma through family/friends, and disaster-related posttraumatic stress disorder and that while directly-exposed individuals were likely to develop posttraumatic stress, indirectly-exposed individuals were more likely to experience depression.

24. Odgers CL, Jensen MR. Annual research review: adolescent mental health in the digital age: facts, fears, and future directions. J Child Psychol Psychiatry. 2020;61(3):336-48. https:// doi.org/10.1111/jcpp.13190.

Publisher's Note Springer Nature remains neutral with regard to jurisdictional claims in published maps and institutional affiliations. 